\title{
STRING THEORY AND MATH: \\ WHY THIS MARRIAGE MAY LAST. MATHEMATICS AND DUALITIES OF QUANTUM PHYSICS
}

\author{
MINA AGANAGIC
}

ABstract. String theory is changing the relationship between mathematics and physics. The central role is played by the phenomenon of duality, which is intrinsic to quantum physics and abundant in string theory.

The relationship between mathematics and physics has a long history. Traditionally, mathematics provides the language physicists use to describe nature, while physics brings mathematics to life: To discover the laws of mechanics, Newton needed to develop calculus. The very idea of a precise physical law, and mathematics to describe it, were born together. To unify gravity and special relativity, Einstein needed the language of Riemannian geometry. He used known mathematics to discover new physics. General relativity has been inspiring developments in differential geometry ever since. Quantum physics impacted many branches of mathematics, from geometry and topology to representation theory and analysis, extending the pattern of beautiful and deep interactions between physics and mathematics throughout centuries.

String theory brings something new to the table: the phenomenon of duality. Duality is the equivalence between two descriptions of the same quantum physics in different classical terms. Ordinarily, we start with a classical system and quantize it, treating quantum fluctuations as small. However, nature is intrinsically quantum. One can obtain the same quantum system from two distinct classical starting points. For every precise question in one description of the theory, there is a corresponding question in the dual description. Duality is similar to a change of charts on a manifold, except it also has the power to map large fluctuations in one description to small fluctuations in the dual, and to relate very hard mathematical problems in one area of mathematics to more manageable ones in another. Dualities are pervasive in string theory. Understanding dualities requires extracting their mathematical predictions and proving the huge set of mathematical conjectures that follow. The best understood duality is mirror symmetry. But mirror symmetry is but one example - many striking dualities have been discovered in quantum field theory (QFT) and many more in string theory over the last 20 years. Duality gives quantum physics, and especially string theory, the power to unify disparate areas of mathematics in surprising ways, and it provides a basis for a long lasting and profound relationship between physics and mathematics 1

\footnotetext{
Received by the editors May 1, 2015.

2010 Mathematics Subject Classification. Primary 00-XX, 81-XX, 51-XX.

${ }^{1}$ Reviews of dualities in string theory can be found in [1]- 3]. For another review of interaction between mathematics and physics, see [4].
} 


\section{KNOT THEORY AND PHYSICS}

To illustrate these ideas, we will pick one particular area of mathematics - knot theory. The central question of knot theory is: When are two knots (or links) distinct? A knot is an oriented closed loop in $\mathbb{R}^{3}$. A link consists of several disjoint, possibly tangled knots. Two knots are considered equivalent if they are homotopic to each other. One approaches the question by constructing knot or link invariants, which depend on the knot up to homotopy.

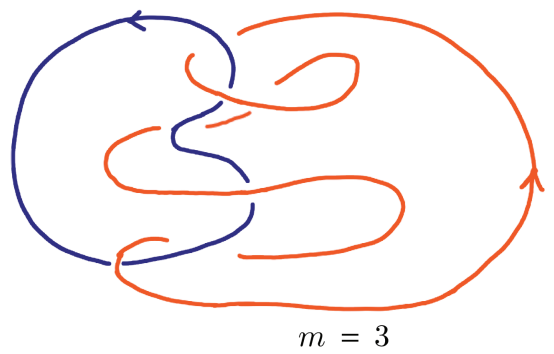

Figure 1. To define the sign of a crossing, we approach a crossing along the bottom strand and assign +1 if the top strand passes from left to right, and -1 otherwise. In the figure, there are six crossings, each contributing +1 , and so $m=3$.

Knot theory was born out of 19th century physics. Gauss's study of electromagnetism resulted in the first link invariant: the Gauss linking number, which is an invariant of a link with two knot components, $K_{1}$ and $K_{2}$. One picks a projection of the link onto a plane and defines (twice) the linking number as the number of crossings, counted with signs:

$$
m\left(K_{1}, K_{2}\right)=\frac{1}{2} \sum_{\operatorname{crossings}\left(K_{1}, K_{2}\right)} \operatorname{sign}(\text { crossing }) .
$$

Gauss discovered the linking number and gave a beautiful integral formula for it:

$$
m\left(K_{1}, K_{2}\right)=\frac{1}{2 \pi} \oint_{K_{1}} \oint_{K_{2}} \frac{\vec{x}_{1}-\vec{x}_{2}}{\left|\vec{x}_{1}-\vec{x}_{2}\right|^{3}} \cdot\left(d \vec{x}_{1} \times d \vec{x}_{2}\right) .
$$

Maxwell discovered it independently, some time later, and noted that it is not a very good link invariant - it is easy to find links that are non-trivial, yet the invariant vanishes. Note that while the first formula (1) for the linking number relies on a choice of a projection, the second one (2) makes it manifest that one is studying a link in three-dimensional space.

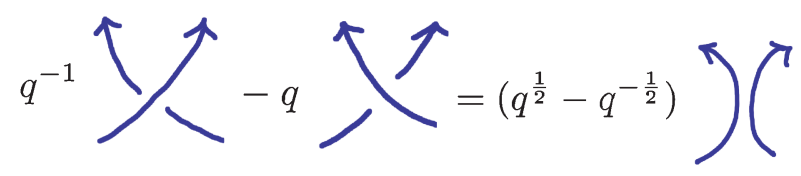

FiguRE 2. Skein relation for the Jones polynomial

Strikingly, quantum physics enters knot theory. In 1984 Vaughan Jones found a very good polynomial invariant of knots and links, by far the best at the time, 
depending on one variable $q$; see [5, 6]. The Jones polynomial is a Laurent polynomial in $q^{\frac{1}{2}}$; it can be computed in a simple way by describing how it changes as we reconnect the strands and change the knot. One picks a planar projection of the knot, a neighborhood of a crossing, and defines the value of the Jones polynomial by the skein relation it satisfies:

$$
q^{-1} J_{K_{+}}-q J_{K_{-}}=\left(q^{\frac{1}{2}}-q^{-\frac{1}{2}}\right) J_{K_{0}},
$$

together with specifying its value for the unknot. While there are examples of distinct knots with the same $J_{K}(q)$, there is no known example of non-trivial knots with $J_{K}(q)$ the same as for the unknot. Despite the ease of construction, the Jones polynomial seems mysterious. Since one has to pick a projection to a plane to define it, it is not obvious at the outset that one obtains an invariant of knots in three-dimensional space; rather this is something one must prove. Secondly, what is the meaning of $q$ ?

Witten discovered that the Jones polynomial has its origin in quantum field theory: Chern-Simons (CS) gauge theory in three dimensions. Like Yang-Mills theory, Chern-Simons theory on a three-manifold $M$ is written in terms of a connection

$$
A=A_{i} d x^{i}
$$

associated with a gauge group $G$. The theory is topological from the outset - its classical action is given in terms of the Chern-Simons form on $M$,

$$
S_{C S}=\frac{1}{4 \pi} \int_{M} \operatorname{Tr}\left(A \wedge d A+\frac{2}{3} A \wedge A \wedge A\right),
$$

and hence it is independent of the choice of metric on $M$. The path integral of the theory

$$
Z(M)=\int \mathcal{D} A \exp \left(i k S_{C S}\right)
$$

where one integrates over spaces of all connections on $M$ and divides by the gauge group, is a topological invariant of $M$. We can introduce a knot $K$ in the theory by inserting an observable line along $K$,

$$
\mathcal{O}_{K}(R)=\operatorname{Tr}_{R} P \exp \left(i \oint_{K} A_{i} d x^{i}\right)
$$

in some representation $R$ of the gauge group ( $P$ denotes path ordering of the exponential). This preserves topological invariance, so

$$
Z(M ; K, R)=\int \mathcal{D} A \exp \left(i k S_{C S}\right) O_{K}(R)
$$

is a topological invariant of the knot $K$ in the three-manifold $M$, which depends only on $G, R$, and $k$. (More precisely, Chern-Simons theory produces an invariant of a framed three-manifold $M$ with framed knots. Framing is a choice of a homotopy class of trivialization of the tangent bundle of $M$ and $K$. The need to fix the framing reflects an ambiguity in the phase of the partition function [7.) The constant $k$ is required to be an integer for the integrand to be invariant under "large" gauge transformations, those corresponding to non-trivial elements of $\pi_{3}(G)$.

Witten made use of the topological invariance of the theory to solve ChernSimons theory exactly on an arbitrary three-manifold $M$ with collection of knots, by cutting the three-manifold into pieces, solving the theory on pieces, and gluing 
them back together. He showed that, taking $M$ to be an $S^{3}, G=S U(2)$, and $R$, the defining two-dimensional representation of $S U(2)$

$$
M=S^{3}, G=S U(2), R=\square,
$$

a suitably normalized Chern-Simons partition function

$$
\left\langle\mathcal{O}_{K}\right\rangle=Z(M ; K) / Z(M ; \bigcirc),
$$

equals the Jones polynomial

$$
\left\langle\mathcal{O}_{K}\right\rangle=J_{K}(q)
$$

The normalization we chose corresponds to setting $J_{\bigcirc}(q)=1$. Chern-Simons theory gave a manifestly three-dimensional formulation of the Jones polynomial. It leads immediately to a vast generalization of Jones's knot invariant by varying the gauge group $G$, the representations, and considering knots in an arbitrary threemanifold $M$. Finally, the relation to Chern-Simons theory showed that the Jones polynomial is a quantum invariant: it is a Laurent polynomial in

$$
q=\exp (i \lambda)
$$

where $\lambda=2 \pi /(k+2)$ plays the role of $\hbar$, the Planck constant, in Chern-Simons theory.

Let us pause for a moment to sketch what it means to say the theory is solvable; cf. [7. It is known that every three-manifold $M$ can be related to $S^{2} \times S^{1}$ by a repeated application of surgery. A surgery to produce from $M$ a new three-manifold proceeds as follows. One picks an imaginary knot in $M$, cuts out its solid torus neighborhood, and glues it back in up to a $U \in S L(2, Z)$ transformation of the boundary. If $U$ is not identity, one obtains a new manifold $M^{\prime}$. Quantum field theory is a functor that associates to a closed three-manifold $M$ a complex number $Z(M)$, the value of the path integral on $M$, and to a manifold with a boundary $B$ a state in the vector space $\mathcal{H}_{B}$, the Hilbert space of the theory based on $B$. Vector spaces associated to the same $B$, with opposite orientation, are canonically dual. Gluing two manifolds over a common boundary $B$ is the inner product of the corresponding states. So surgery on three-manifolds translates to the following statement in QFT:

$$
Z\left(M^{\prime}\right)=\left\langle 0 \mid M^{\prime} / K\right\rangle=\langle 0|U| M / K\rangle .
$$

Here $\langle 0|$ is the state corresponding to a solid torus with no insertions, and $\left|M^{\prime} / K\right\rangle$ is the state corresponding to $M^{\prime}$ with a neighborhood of the knot $K$ cut out. An arbitrary state in $\mathcal{H}_{T^{2}}$ can be obtained from a solid torus with an observable line colored by a representation $R$ running through it. If we denote the resulting state $\langle R|$, we can write

$$
\langle 0| U=\sum_{R}\langle R| U_{0 R}
$$

The sum runs over a finite set of representations of $G$, depending on $k$. (The Hilbert space $\mathcal{H}_{B}$ of Chern-Simons theory with gauge group $G$ at level $k$ is the same as the space of conformal blocks of $G_{k}$ WZW model; the latter is finite dimensional for any B.) This implies

$$
Z\left(M^{\prime}\right)=\sum_{R}\langle R \mid M / K\rangle U_{0 R}=\sum_{R} Z\left(M^{\prime}, K, R\right) U_{0 R},
$$

where $Z\left(M^{\prime}, K, R\right)$ corresponds to the partition function on $M^{\prime}$ with an actual knot $K$ colored by representation $R$ running through it. In this way, by repeated 
surgeries, we can reduce any three-manifold invariant to that of $S^{2} \times S^{1}$ with a braid running along the $S^{1}$. In turn, the latter can be computed by

$$
Z\left(S^{2} \times S^{1}, L, R_{i}\right)=\operatorname{Tr}_{\mathcal{H}_{S^{2}, R_{i}}} B_{L},
$$

which comes about by first cutting the $S^{2} \times S^{1}$ open into $S^{2} \times R$, straightening the braid out, and then recovering the original braid by finding a collection $B_{L}$ of time-ordered diffeomeorphisms of a sphere $S^{2}$ with marked points, which rebraids the braid. Gluing the ends together corresponds to taking the trace of $B_{L}$, acting on the Hilbert space $\mathcal{H}_{S^{2}, R_{i}}$ of the theory on $S^{2}$ with marked points colored by representations $R_{i}$ determined by the braid.

To solve the theory, one needs only a finite set of data. The $S L(2, \mathbb{Z})$ transformations of the torus are generated by a pair of matrices, $S$ and $T$, satisfying

$$
S^{4}=1, \quad(S T)^{3}=S^{2},
$$

representing the action of $S L(2, \mathbb{Z})$ on $\mathcal{H}_{T^{2}}$. Similarly, the brading matrix $B_{L}$ is obtained from a finite set of data, the braiding matrix $B$ and fusion matrix $F$ on a four-punctured sphere [8]. For Chern-Simons theory based on gauge group $G$, at level $k$, the $S, T, B$, and $F$ are finite-dimensional matrices acting on conformal blocks of $G_{k}$ WZW 2d CFT. Reshetikhin and Turaev formalized this in terms of modular tensor categories [8]. Thus, one can reduce finding knots and three-manifold invariants for arbitrary $G$ and $k$ and representations $R$ to matrix multiplication of a small set of matrices.

\section{Gromov-Witten theory}

Quantum physics enters modern mathematics in other places as well. GromovWitten theory is an example. There, one studies quantum intersection theory of a projective variety $X$ (see [9] for a review and [10] for a quick overview). Classical intersection corresponds to picking classes

$$
\gamma_{1}, \ldots, \gamma_{n} \in H_{*}(X)
$$

with degrees $\sum_{i} \operatorname{deg}\left(\gamma_{i}^{\vee}\right)=2 d$, where $d=\operatorname{dim}_{\mathbb{C}}(X)$, and computing their intersection numbers, counted with signs

$$
\left\langle\gamma_{1}, \ldots, \gamma_{n}\right\rangle_{0,0}=\int_{X} \gamma_{1}^{\vee} \wedge \cdots \wedge \gamma_{n}^{\vee}
$$

where $\gamma_{i}^{\vee} \in H^{*}(X)$ denotes the Poincaré dual of $\gamma_{i}$. Enumerative geometry turns this into a deeper geometric question by counting intersections over algebraic curves, insead over points: one would like to know how many algebraic curves of a given degree $\beta \in H_{2}(X)$ and genus $g$ meet at $\gamma_{1}, \ldots, \gamma_{n}$ points. The corresponding invariant

$$
\left\langle\gamma_{1}, \ldots, \gamma_{n}\right\rangle_{g, \beta}
$$

can be defined by picking a curve $\Sigma$ of genus $g$, with $n$ marked points $p_{1}, \ldots, p_{n}$, and considering intersection theory on the moduli space $\mathcal{M}_{g, n}(X, \beta)$ of holomorphic maps

$$
\phi: \Sigma \rightarrow X
$$

of degree $d$. More precisely, as explained by Kontsevich, one needs to consider the moduli space of stable maps $\overline{\mathcal{M}}_{g, n}(X)$. This is a compactification of $\mathcal{M}_{g, n}(X, \beta)$ by allowing the domain curve $\Sigma$ to have "ears", which are additional $S^{2}$ that bubble off, and by considering stable maps, which he defined. Imposing the incidence condition 
that $\phi\left(p_{i}\right) \subset \gamma_{i}$ is implemented by pulling back the Poincaré dual class $\gamma_{i}^{\vee}$ via the evaluation map $\mathrm{ev}_{i}$, the evaluation map maps a point in the moduli space of maps to $\phi\left(p_{i}\right)$ :

$$
\left\langle\gamma_{1}, \ldots, \gamma_{n}\right\rangle_{g, d}=\int_{\left[\overline{\mathcal{M}}_{g, n}(X, \beta)\right]} \operatorname{ev}_{1}^{*}\left(\gamma_{1}^{\vee}\right) \cdots \mathrm{ev}_{n}^{*}\left(\gamma_{n}^{\vee}\right),
$$

where the brackets [...] denote the (virtual) fundamental class. For genus zero, degree zero curves, the definition agrees with the classical intersection numbers in (3). At genus zero, it is natural to combine the classical answer (3) and the higher degree data into a generating function of quantum intersection numbers of $X$,

$$
\left\langle\gamma_{1}, \ldots, \gamma_{n}\right\rangle_{0, Q}=\sum_{\beta \in H_{2}(X)}\left\langle\gamma_{1}, \ldots, \gamma_{n}\right\rangle_{0, \beta} Q^{\beta}
$$

For a map of degree $\beta$ to $X, Q^{\beta}$ is the exponent the area of the target curve, $Q^{\beta}=\exp \left(-\int_{\Sigma} \phi^{*} \omega\right)$, where $\omega$ is the Kähler form on $X$. The leading term in the series is the classical intersection, and the subleading terms are quantum corrections to it.

\subsection{Gromov-Witten theory and topological string theory. Gromov-Witten} theory originates from string theory. It computes the amplitudes of a topological variant of superstring theory, called the A-model topological string.

In quantum field theory to describe a particle propagating on a manifold $X$, one sums over all maps from graphs $\Gamma$ to $X$, satisfying certain conditions, where one allows moduli of graphs to vary. In string theory we replace point particles by strings, the maps from graphs $\Gamma$ by maps from Riemann surfaces $\Sigma$ to $X$. In superstring theory, one formulates this in terms of a path integral of a supersymmetric $2 \mathrm{~d}$ QFT on $\Sigma$, describing a string propagating on $X$. To get topological string theory, one modifies the supersymmetry generator $Q$ to square to zero, $Q^{2}=0$, on arbitrary $\Sigma$. This turns the $2 \mathrm{~d}$ QFT into a topological quantum field theory on $\Sigma$ of cohomological type, with differential $Q$. The world sheet path integral receives contributions only from configurations that are annihilated by $Q$. If $X$ is a Calabi-Yau manifold, there are two inequivalent ways to obtain a TQFT, leading to topological A- and B-model string theories. They correspond to two distinct generators, $Q_{A}$ and $Q_{B}$, each satisfying $Q_{A, B}^{2}=0$. Topological A-type string theory exists for any Kähler manifold $X$. Restricting to configurations annihilated by $Q_{A}$ turns out to restrict one to studying holomorphic maps to $X$ only, leading to Gromov-Witten theory.
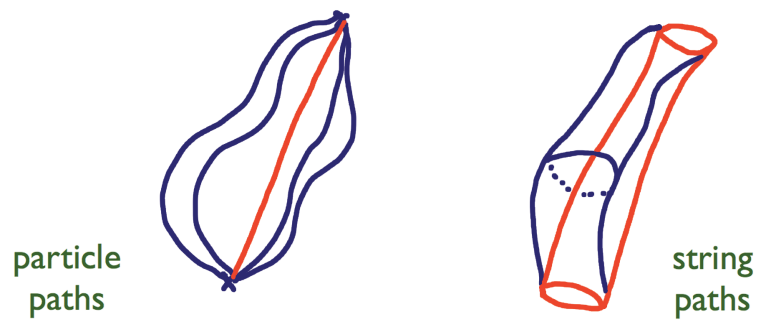

FIGURE 3. In string theory one sums over all possible paths of a string, leading to sum over surfaces. 
In the B-model, the maps annihilated by $Q_{B}$ are the constant maps, resulting in a simpler theory, depending on complex structure of $X$ only.

Topological string theory was introduced by Witten in [12,13] and developed by many (see for example [14,15] and 9] for a review). The mathematical formulation of Gromov-Witten theory is due to Kontsevich, Manin, Fukaya, and many others [9. The development of Gromov-Witten theory is an example of new mathematics that is inspired by questions in physics.

\section{Duality}

A quantum system is described by a collection of observables $\mathcal{O}_{i}$, corresponding to physical quantities in the theory, and expectation values of these observables,

$$
\left\langle\mathcal{O}_{1} \cdots \mathcal{O}_{n}\right\rangle
$$

which physicists call amplitudes, or correlation functions. In Chern-Simons theory, the observables ended up associated to knots in a three-manifold $M$, colored by representations $R$ of the gauge group; in Gromov-Witten theory, the observables were related to homology classes $\gamma_{i}$ in $X$.

To find the correlation functions, one starts with a classical limit of the system and a quantization procedure. In Gromov-Witten theory of $X$, one would start with a two-dimensional topological theory on a genus $g$ Riemann surface $\Sigma$, based on maps to $X[9]$. The description we are giving assumes quantum fluctuations are small. This opens up a possibility for the same physical system to have different descriptions with different starting classical points, yet which result in the same set of quantum amplitudes. This expresses the fact that physics is intrinsically quantum - only our descriptions of it rely on classical limits, and the classical limits need not be unique. The map between the two descriptions of the single physical system is called a duality.

3.1. Mirror symmetry. Perhaps the best known example of a duality is mirror symmetry. Mirror symmetry relates a topological A-model string on a CalabiYau $X$ to a topological B-type string theory on the mirror Calabi-Yau $Y$ (the phenomenon was discovered in [16]; for a review see [9]). The underlying CalabiYau manifolds are different, even topologically, as mirror symmetry reflects the hodge diamond

$$
h^{p, q}(X)=h^{d-p, q}(Y),
$$

yet the A-model on $X$ and the B-model on $Y$ are the same quantum theory. Here $d$ is the complex dimension of $X$ and $Y$. The amplitudes of the A-type topological string are computed by Gromov-Witten theory. The B-type topological string reduces to a quantum field theory on $Y$ which quantizes the variations of complex structures; this is related to the fact that $Q_{B}$ vanishes on constant maps. In particular, the $g=0$ amplitudes can be read off from classical geometry. The most interesting case is $d=3$; otherwise, many amplitudes vanish on dimension grounds. This can be seen from the formula for the (virtual) dimension of the moduli space of stable maps in Gromov-Witten theory:

$$
\operatorname{dim}_{\mathbb{C}} \overline{\mathcal{M}}_{g, n}(X, \beta)=-\beta \cdot c_{1}(T X)+(1-g)(d-3)+n .
$$

In the Calabi-Yau case, per definition, $c_{1}(T X)$ vanishes in cohomology; for $d=3$, the moduli space has positive dimension for any $g, d$, and $n$. 
The first prediction of mirror symmetry is that the genus zero amplitudes on $X$ and $Y$ agree. On $X$, computing this leads to quantum intersection numbers: choosing $\gamma_{i}, \gamma_{j}, \gamma_{k}$ to be three divisors in $X, \gamma_{i, j, k}^{\vee} \in H^{2}(X)$, one computes

$$
\left\langle\gamma_{i}, \gamma_{j}, \gamma_{j}\right\rangle_{0, Q}=\sum_{\beta \in H_{2}(X)}\left\langle\gamma_{i}, \gamma_{j}, \gamma_{k}\right\rangle_{0, \beta} Q^{\beta}
$$

The $\beta=0$ term in the sum is the classical intersection number of the three divisor classes, and subsequent terms involve intersection theory on the moduli space of stable maps, as we described above. In the mirror B-model on $Y$, the entire sum is captured [17] by classical geometry of $Y$ :

$$
\left\langle\gamma_{i}, \gamma_{j}, \gamma_{j}\right\rangle_{0, Q}=\int_{Y} \Omega \wedge \frac{\partial}{\partial t_{i}} \frac{\partial}{\partial t_{j}} \frac{\partial}{\partial t_{k}} \Omega
$$

This leads to a striking simplification. Here, $\Omega \in H^{(3,0)}(Y)$ is the unique holomorphic volume form on $Y$, whose existence is guaranteed by the Calabi-Yau condition. The parameters $t_{i}$ are suitably chosen moduli of complex structures on $Y$.

The higher genus amplitudes in the B-model quantize the variations of complex structures on $Y$. In complex dimension 3, the theory one gets is the "KodairaSpencer theory of gravity", formulated in [15. The study of a B-model in other dimensions was initiated in [18].

3.2. Large $N$ duality. A duality, discovered by Gopakumar and Vafa [20, relates $G=U(N)$ Chern-Simons theory at level $k$, on

$$
M=S^{3},
$$

with an A-model topological string on

$$
X_{\mathbb{P}^{1}}=\mathcal{O}(-1) \oplus \mathcal{O}(-1) \rightarrow \mathbb{P}^{1} .
$$

To complete the statement of the duality conjecture, we need to explain the map of parameters and the correspondence of observables. In defining Chern-Simons theory on the $S^{3}$, we get to chose two parameters, the integers $N$ and $k$. The Gromov-Witten theory on $X_{\mathbb{P} 1}$ depends on the size of the $\mathbb{P}^{1}$,

$$
t=\int_{\mathbb{P}^{1}} \omega
$$

and $\lambda$, the genus counting parameter. The latter enters if, instead of fixing the genus of the Riemann surface $g$ as we did previously, we want to form a generating function by summing over $g$. The duality maps the parameters of Chern-Simons theory to the parameters of Gromov-Witten as follows:

$$
t=\frac{2 \pi N}{k+N}, \quad \lambda=\frac{2 \pi}{k+N} .
$$

The first prediction of the duality is the equivalence of partition functions before we introduce knots in $S^{3}$ :

$$
Z_{C S}=Z_{G W}
$$

The Chern-Simons partition function on the $S^{3}$ can be computed as the matrix element of the $S$ matrix (acting on $S U(N)_{k}$ WZW model on $T^{2}$ )

$$
Z_{C S}\left(S^{3}\right)=\langle 0|S| 0\rangle=S_{00}
$$


This is an example of obtaining a three-manifold, in this case $M^{\prime}=S^{3}$, from $M=S^{2} \times S^{1}$ by surgery. We start by excising a neighborhood of an unknot in $S^{2} \times S^{1}$ running around the $S^{1}$ and at a point on $S^{2}$, which splits $M$ into two solid tori. To recover $S^{2} \times S^{1}$, we simply glue the solid tori back together, with trivial identification $U=1$; to obtain an $S^{3}$ instead, we glue them with an $S$ transformation of the $T^{2}$ boundary.

The Gromov-Witten partition function $Z_{G W}$ is defined as the generating function of all maps to $X_{\mathbb{P}^{1}}$ with no insertions:

$$
Z_{G W}\left(X_{\mathbb{P}^{1}}\right)=\exp \left(\sum_{g=0}\langle 1\rangle_{g, Q} \lambda^{2 g-2}\right)=\exp \left(\sum_{g=0, \beta \in H_{2}(W)}\langle 1\rangle_{\beta, g} Q^{\beta} \lambda^{2 g-2}\right),
$$

where

$$
\langle 1\rangle_{\beta, g}=\int_{\left[\overline{\mathcal{M}}_{g, 0}(X, \beta)\right]} 1
$$

and $Q^{\beta}=\exp (-\beta t)$. In this case the degree of the curve is captured by a single number, since $X_{\mathbb{P} 1}$ has a single non-trivial two-cycle class corresponding to the $\mathbb{P}^{1}$ itself. The Gromov-Witten partition function of $X_{\mathbb{P}}^{1}$ was computed by Faber and Pandharipande in [21] by computing $\langle 1\rangle_{g, Q}$ for every $g$. The Chern-Simons partition function is known, since the $S$ matrix is known explicitly. Gopakumar and Vafa 20] showed that $Z_{C S}\left(S^{3}\right)$ equals $Z_{G W}\left(X_{\mathbb{P 1}}\right)$ by explicit computation. It is striking that the one sums up infinitely many Gromov-Witten invariants in a single matrix element $S_{00}$ in Chern-Simons theory.

The observables of Chern-Simons theory correspond to line operators associated to knots $K$ colored by irreducible representations $R$ of $G$. Introducing knots on $S^{3}$ corresponds on $X_{\mathbb{P}^{1}}$ to allowing maps to have boundaries on a Lagrangian submanifold $L_{K}$ in $X_{\mathbb{P}^{1}}$, where $L_{K}$ gets associated to a knot $K$ in a precise way [22. If we have several knots on $S^{3}$, one will introduce a corresponding Lagrangian for each knot. To explain how these Lagrangians are constructed [23], we must first explain the origin of the duality.

3.2.1. Chern-Simons theory as a string theory. $S U(N)$ Chern-Simons theory on a three-manifold $M$ turns out to compute open topological A-model amplitudes on

$$
X_{M}=T^{*} M,
$$

the total space of the cotangent bundle on $M$. One takes the A-model topological string on $X_{M}$, but considers maps with boundaries on $M$ :

$$
\phi: \Sigma \rightarrow X_{M}, \quad \partial \Sigma \rightarrow M .
$$

Allowing boundaries corresponds to considering open topological A-models. More precisely, we formally need to take $N$ copies of $M$ in $X_{M}$ and keep track of which copy of $M$ a given component of the boundary of $\Sigma$ falls onto. As in the closed case, only the holomorphic maps end up contributing to amplitudes. In fact, as there are no finite holomorphic curves of any kind in $X_{M}=T^{*} M$, only degenerate maps contribute - those where the image curves degenerate to graphs on $M$. Witten showed that the resulting graph expansion is the Feynman graph expansion of $S U(N)$ Chern-Simons theory. This means that Chern-Simons theory on $M$ computes open topological string amplitudes in this background in the same way 
Gromov-Witten theory on $X$ computes closed A-model topological string amplitudes on $X$. A mathematical consequence of this is that the $G=S U(N)$ ChernSimons partition function on $M$ must have

$$
Z_{C S}(M)=\exp \left(\sum_{g, h=0} F_{g, h}^{C S} N^{h} \lambda^{2 g-2+h}\right),
$$

where $F_{g, h}^{C S}$ are numbers independent of $N, k$, which capture contributions of maps from surfaces $\Sigma$ that have genus $g$ and $h$ boundary components. For every boundary we have a choice of which copy of $M$ it falls on, hence the power $N^{h}$, and $\lambda$ keeps track of the Euler characteristic of such as a surface which equals $2-2 g-h$. The numbers $F_{g, h}$ (the perturbative Chern-Simons invariants) play a role in knot theory [24,25] - they are related to Vasilliev invariants and to the Kontsevich integral.

Observables in Chern-Simons theory on $M$ are associated to knots. Introducing a knot $K$ in $U(N)$, Chern-Simons theory on $M$ corresponds, in the topological A-model on $X_{M}$, to introducing a Lagrangian $L_{K}$ which is a total space of the conormal bundle to the knot $K$ in $T^{*} M[22$. For every point $P$ on the knot $K$ in $M$, one takes the tangent vector to the knot, and defines a rank 2 subbundle of the cotangent bundle, by taking all cotangent vectors that vanish on it. The conormal condition implies that $L_{K}$ is Lagrangian; this in turn guarantees that the adding boundaries preserve topological invariance of the A-model. Instead of fixing the representation $R$ coloring the knot, it is better to sum over representations and consider a formal combination of observables

$$
\mathcal{O}_{K}(U)=\sum_{R} \mathcal{O}_{K}(R) \operatorname{Tr}_{R} U
$$

where $U$ is an arbitrary unitary matrix of rank $m$, and the sum runs over arbitrary irreducible representations of $U(N)$. The choice of rank $m$ is the number of copies of $L_{K}$ we take (similar to the way we took $N$ copies of $M$ to get $S U(N)$ ChernSimons theory). This observable probes representations $R$ whose Young diagram has no more than $m$ rows, since otherwise $\operatorname{Tr}_{R} U$ vanishes. Computing the ChernSimons partition function in the presence of knot $K$ with this observable inserted corresponds to studying the A-model on $X_{S^{3}}$ where one allows boundaries on $L_{K}$. The eigenvalues $\left(u_{1}, \ldots, u_{m}\right)$ of $U$ keep track of which of the $m$ copies of $L_{K}$ the boundary component lands on: a single boundary on the $i$ th copy of $L_{K}$ gets weighted by $u_{i}$. The resulting partition function is a symmetric polynomial of the $u$ 's (using the relation between $S_{m}$ symmetric polynomials and characters of $U(m)$ in various representations) reflecting the $S_{m}$ permutation symmetry of $m$ copies of $L_{K}$. The open topological A-model is expected to have the same relation to open Gromov-Witten theory, as the closed A-model has to closed Gromov-Witten theory, where "closed" refers to absence of boundaries of the domain curves $\Sigma$. Unlike the closed Gromov-Witten theory, the foundations of the open Gromov-Witten theory are not entirely in place yet, although progress is being made [26].

3.2.2. Large $N$ duality is a geometric transition. Gopakumar and Vafa conjectured that large $N$ duality has a geometric interpretation as a transition that shrinks the $S^{3}$ and grows the $\mathbb{P}^{1}$ :

$$
X_{S^{3}} \quad \rightarrow \quad X_{*} \quad \rightarrow \quad X_{\mathbb{P}^{1}} .
$$

In the geometric transition, the $S^{3}$ disappears and with it the boundaries of maps. If the conjecture is true, it leads to an extraordinary insight: the transition changes 
topology of the manifolds classically and becomes a change of description - the theories on $X_{S^{3}}$ (in the presence of boundaries on $N$ copies of the $S^{3}$ ) and on $X_{\mathbb{P}^{1}}$ are the same. The passage from one description to the other is perfectly smooth, like a change of charts on a single manifold.
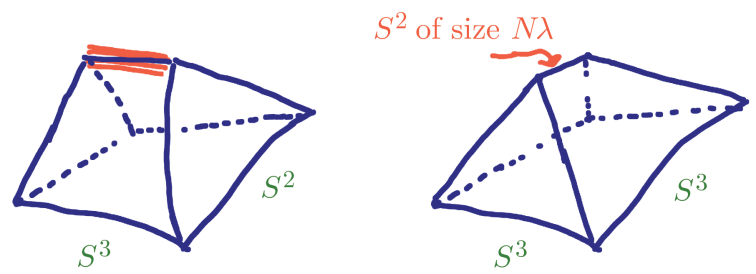

FIgURE 4. Large $N$ duality is a geometric transition that shrinks the $S^{3}$ and grows an $S^{2}$ of size $t=N \lambda$.

When, $N$ becomes large, it is natural to sum over $h$ in (8): while $\lambda$ still keeps track of the Euler characteristic of the underlying Riemann surface, the explicit $N$ dependence disappears. This reflects the fact that both the boundaries and the $S^{3}$ disapear in the large $N$ dual description. The large $N$ duality implies that

$$
\sum_{h} F_{g, h}^{C S} t^{h}=F_{g}^{G W}(t), \quad t=N \lambda,
$$

which is what Gopakumar and Vafa proved in [20] by showing $Z_{C S}=Z_{G W}$. We defined $F_{g}^{G W}(t)$ by

$$
Z_{G W}\left(X_{\mathbb{P}^{1}}\right)=\exp \left(\sum_{g} F_{g}^{G W} \lambda^{2 g-2}\right)
$$
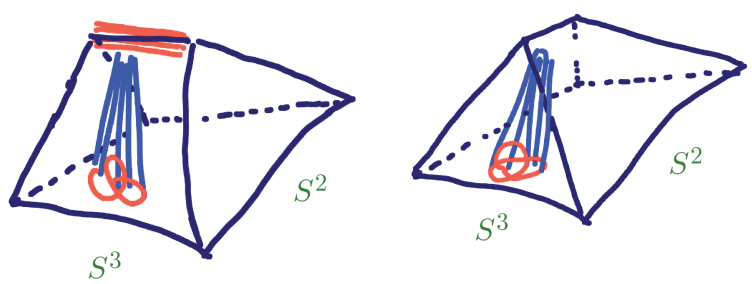

Figure 5. The Lagrangian $L_{K}$ conormal to a knot $K$ in $S^{3}$ gets pushed through the transition.

The quantum knot invariants of $K$ are computed by studying an open topological A-model on $X_{S^{3}}$ where one allows boundaries on the Lagrangian $L_{K}$. The geometric interpretation of the large $N$ duality as a transition between $X_{S^{3}}$ and $X_{\mathbb{P}^{1}}$ helps us identify what this corresponds to on the dual side. The asymptotic geometry of $X_{S^{3}}$ and $X_{\mathbb{P}^{1}}$ are the same - they both approach cones over $S^{3} \times S^{2}$ - they are just filled in differently in the interior. If we first lift $L_{K}$ off the $S^{3}$, it can go through the geometric transition smoothly to become a Lagrangian on $X_{\mathbb{P}^{1}}$, which we will denote $L_{K}$ again. In particular, the topology of the Lagrangian is the same, $\mathbb{R}^{2} \times S^{1}$, both before and after the transition. The construction was made precise in 23 . 
This leads to a generalization of the basic relation (10) as follows. One considers the partition function of Chern-Simons theory with observable (9) inserted:

$$
Z_{C S}\left(S^{3}, K, U\right)=\left\langle\mathcal{O}_{K}(U)\right\rangle \text {. }
$$

This has expansion

$$
Z_{C S}\left(S^{3}, K, U\right)=\exp \left(\sum_{p} \sum_{g, h, k_{1}, \ldots, k_{p}=0} F_{g, h, k_{1}, \ldots, k_{p}}^{C S} N^{h} \lambda^{2 g-2+h+p} \operatorname{Tr} U^{k_{1}} \cdots \operatorname{Tr} U^{k_{p}}\right) .
$$

The large $N$ duality conjecture states that one can sum over $h$ to get

$$
Z_{G W}\left(X_{\mathbb{P}^{1}}, L_{K}, U\right)=\exp \left(\sum_{p} \sum_{g, k_{1}, \ldots, k_{p}=0} F_{g, k_{1}, \ldots, k_{p}}^{G W}(t) \lambda^{2 g-2+p} \operatorname{Tr} U^{k_{1}} \cdots \operatorname{Tr} U^{k_{p}}\right) ;
$$

in other words that

$$
\sum_{h} F_{g, h, k_{1}, \ldots, k_{p}}^{C S} t^{h}=F_{g, k_{1}, \ldots, k_{p}}^{G W}(t)
$$

For simple knots and links - the unknot and the Hopf-link - one is able to formulate a computation of the open topological A-model amplitude on $X_{\mathbb{P}}^{1}$ directly in open Gromov-Witten theory [27, 28] and verify the conjecture. For more complicated knots, one needs substantial progress in formulating the open Gromov-Witten side to be able to test the predictions.

The large $N$ duality relating $S U(N)$ Chern-Simons theory to closed string theory is a part of a family of dualities, whose existence was conjectured by 't Hooft in the 1970s [29. He showed that $S U(N)$ gauge theories on general grounds always have a Feynman graph expansion of the form (8) with coefficients $F_{g, h}$ that depend on the theory, but not on $N$ or $\lambda$. As a consequence, whenever $N$ becomes large, it is natural to re-sum the perturbative series. The result has the form of a closed string Feynman graph expansion; the main question is to identify the dual closed string theory. For Chern-Simons theory on $S^{3}$, the closed string theory is GromovWitten theory on $X_{\mathbb{P}^{1}}$. Whenever it exists, the closed string description gets better and better the larger $N$ is, hence the name.

3.3. Gromov-Witten/Donaldson-Thomas correspondence. One is used to studying Gromov-Witten theory by fixing the genus of the Riemann surface $\Sigma$. However, Chern-Simons theory and large $N$ duality suggest it is far more economical to consider all genera at once: Chern-Simons amplitudes are simplest when written in terms of $q=\exp (i \lambda)$ rather than $\lambda$. More generally, the fact that one can sum up perturbation series in gauge theory and solve the theory exactly (at least in principle) is one of the main reasons why large $N$ duality plays such an important role in physics: gauge theory allows one to circumvent the usual genusby-genus formulation of closed string theory.

For Gromov-Witten theory on toric Calabi-Yau threefolds, the theory was indeed solved in this way. Using ideas that originated from large $N$ dualities and Chern-Simons theory, [30] conjectured a solution of Gromov-Witten theory on any toric Calabi-Yau threefold by cutting up the Calabi-Yau into $\mathbb{C}^{3}$ pieces, solving the theory on $\mathbb{C}^{3}$ exactly and giving a prescription for how to glue the solution on pieces to a solution of the theory on $X$. The result is the topological vertex formalism for Gromov-Witten theory of toric Calabi-Yau manifolds, which expresses the partition function for a fixed degree $\beta \in H_{2}(X)$ in terms of rational functions 
of $q$ [30]. The topological vertex conjecture was proven by Maulik, Oblomkov, Okounkov, and Pandharipande in [28, who also generalized it away from Calabi-Yau manifolds to arbitrary toric threefolds.

The resulting invariants of toric threefolds turn out to be directly captured by a precise mathematical theory - the Donaldson-Thomas theory of $X$. The theory was introduced in [31, and Okounkov, Maulik, Pandharipande, and others provided its foundations 32,33 . The fact that they are also related to Gromov-Witten invariants of $X$ is the content of the Gromov-Witten/Donaldson-Thomas correspondence [32,33. Mathematically, Donaldson-Thomas theory also deals with counting Riemann surfaces in $X$ - but it does so in a very different way than Gromov-Witten theory. Instead of describing parameterized curves in $X$ in terms of holomorphic maps $\phi: \Sigma \rightarrow X$ as one does in Gromov-Witten theory, in Donaldson-Thomas theory one describes curves by algebraic equations (see [10] for a review). Let $X$ be a projective variety, let $X \subset \mathbb{P}^{r}$ for some $r$, and let $z_{i}$ be the homogenous coordinates of $\mathbb{P}^{r}$. We can describe the curve $C$ in $X$ as the locus of a set of homogenous polynomials

$$
f(z)=0
$$

which vanish on $C$. The set of all such functions form an ideal $I(C)$ inside $\mathbb{C}\left[z_{0}, \ldots, z_{r}\right]$. We fix the class $\beta \in H_{2}(X)$ and $\chi$, the holomorphic Euler characteristic of $C(\chi=1-g$, where $g$ is the arithmetic genus of $C)$, and denote the moduli space of $C$ by $\mathrm{I}(X, \beta, \chi)$. The moduli space is isomorphic to the Hilbert scheme of curves in $X . X$ being a threefold is special in this case too: the resulting simplifications allow one to construct a (virtual) fundamental cycle in $\mathrm{I}(X, \beta, \chi)$, denoted by $[\mathrm{I}(X, \beta, \chi)]$. The analogue of (4) is

$$
\left\langle\gamma_{1}, \ldots, \gamma_{n}\right\rangle_{\beta, \chi}=\int_{[I(X ; \beta, \chi)]} c_{2}\left(\gamma_{1}\right) \cdots c_{2}\left(\gamma_{n}\right) .
$$

To construct $c_{2}(\gamma)$ takes a special sheaf, the universal ideal sheaf $J, J \in \mathrm{I}(X) \times X$ which has the property that $c_{2}(J)$ is the locus in $\mathrm{I}(X) \times X$ corresponding to the set (ideal $I$, point of curve determined by $I) . c_{2}(\gamma)$ is the locus of curves meeting $\gamma$-this is the coefficient of $\gamma^{\vee}$ in the decomposition of $c_{2}(J) \in H^{2}(\mathrm{I}(X) \times X)$. Let

$$
Z_{D T}(\gamma, q)_{\beta}=\sum_{\chi}\left\langle\gamma_{1}, \ldots, \gamma_{n}\right\rangle_{\beta, \chi}^{D T} q^{\chi}
$$

The conjecture equates this, up to normalization, with

$$
Z_{G W}(\gamma, \lambda)_{\beta}=\sum_{\chi}\left\langle\gamma_{1}, \ldots, \gamma_{n}\right\rangle_{\beta, g}^{G W} \lambda^{2 g-2} .
$$

(In this section we allow disconnected domain curves, as this is the natural thing to do if we want to glue the theory on $X$ from pieces. This is also why we do not exponentiate the right-hand sides.) More precisely,

$$
(-\lambda)^{-\operatorname{vdim}} Z_{G W}^{\prime}(\gamma ; \lambda)_{\beta}=(-q)^{-\operatorname{vdim} / 2} Z_{D T}^{\prime}(\gamma ; q)_{\beta},
$$

where $q=\exp (i \lambda)$ and ' denotes dividing by contributions of degree zero curves, which we do on both sides. The Donaldson-Thomas partition function has a beautiful statistical-mechanics interpretation in terms of counting boxes stacked up in the toric base of $X$. One sums over a set of box configurations obeying certain natural conditions and weighs the sum with $q^{\# \text { boxes }}$. Remarkably, the box-counting problem has a saddle point as $q \rightarrow 1$ and $\lambda \rightarrow 0$. In this limit, the cost of adding 
a box is small and a limiting shape develops that dominates the partition function $Z_{D T}$ in the limit. Strikingly, the limiting shape encodes the geometry of the Calabi-Yau $Y$ mirror to $X$ [34,35].

The duality relating Gromov-Witten theory and Donaldson-Thomas theory has a physical interpretation in M-theory, a quantum theory that underlies and unifies all string theories [36. Despite the simple appearance - relating counting curves in two different ways - the duality that underlies the Gromov-Witten/DonaldsonThomas correspondence is far from trivial. In particular, Donaldson-Thomas theory leads to many generalizations that go beyond Gromov-Witten theory. In particular, Donaldson-Thomas theory explains the mysterious integrality of GromovWitten invariants which was noticed very early on: while one can express GromovWitten invariants in terms of a set of integers, this is not manifest from the definition of the theory - Gromov-Witten theory naturally leads to counts of curves which are rational numbers, not integers, since the underlying moduli spaces are not smooth. One expects that relation of Donaldson-Thomas and Gromov-Witten theories is much like the diagram in Figure 7 -there is a large parameter space of Donaldson-Thomas theory, the tips of which have Gromov-Witten interpretation.

\section{Combining DUAlities And KnOt theory}

Duality is like a change of charts on a manifold; in particular, we can combine dualities, and get even more mileage from them. For example, one can combine large $N$ duality and mirror symmetry. It turns out that this can shed fundamentally new light on knot theory, but and explain this we need to back up and explain the origin of mirror symmetry first.

4.1. Homological mirror symmetry and the SYZ conjectures. We have seen that Gromov-Witten theory computes quantum corrections to the classical geometry of a Calabi-Yau $X$. Mirror symmetry sums up these corrections, in terms of the geometry of the mirror Calabi-Yau $Y$. One can make this precise and give a (conjectural) description for how the classical geometry of $Y$ emerges from quantum geometry of $X$.

There are two mathematical conjectures that capture aspects of mirror symmetry. The homological mirror symmetry conjecture of Kontsevich [37] relates categories of allowed boundary conditions of a topological A-model on $X$ and a topological B-model on $Y$. The former is captured by the Fukaya category $D^{\mathcal{F}}(X)$ of $(X, \omega)$ whose objects are Lagrangian submanifolds $L \subset X$ equipped with a unitary flat connection $A$ :

$$
\left.\omega\right|_{L}=0, \quad F=0,
$$

where $F=d A$ is the curvature of the flat connection $A$. Holomorphic maps $\phi$ : $\sigma \rightarrow X$ are allowed to have boundaries on $L \in X$; the connection on $L$ couples to the boundaries. We have seen examples of this, when $X=T^{*} M$, where we took $L=M$ or $L_{K}$, the Lagrangian associated to the knot. The $A$ connection on $M$ is the Chern-Simons connection. The morphisms in the category are associated to strings with endpoints on pairs of Lagrangians. Kontsevich conjectured that on the mirror $Y$ there is an equivalent category, the bounded derived category of coherent sheaves, $D^{b}(Y)$. The homological mirror symmetry conjecture was recently proven for a famous example of the quintic Calabi-Yau manifold $X$ and its mirror 38. 
Among the objects in $D^{b}(Y)$, a privileged role is played by the structure sheaf $\mathcal{O}_{p}$ for $p$ a point in $Y$. The moduli space of $\mathcal{O}_{p}$ is $Y$ itself. Mirror symmetry implies that there must be an object in the Fukaya category of $X$ with the same moduli space. Strominger, Yau, and Zaslow 39] showed that this fact alone implies that the mirror pair of manifolds $(X, Y)$ must both be $T^{3}$ fibrations over a common base $B$, with fibers that are (special) Lagrangian tori. Let $X$ be a $T^{3}$ fibration,

$$
T^{3} \rightarrow X \rightarrow B
$$

over a base $B$, and let $L_{p}$ be a $T^{3}$ fiber of $X$ above a point in $p \in B$. The moduli space of $L_{p}$ is the base $B$ itself. The full moduli space is a fibration over this, by the moduli of a flat $U(1)$ bundle on $T^{3}$. The moduli of a $U(1)$ bundle on $T^{3}$ is the dual torus $\hat{T}^{3}$. More precisely, the resulting moduli can get corrected by "disk instantons" (maps from the disk to $X$ with boundaries on $L$ ), and taking this into account results in the mirror manifold

$$
\hat{T}^{3} \rightarrow Y \rightarrow B .
$$

This is the $S Y Z$ mirror symmetry conjecture. This gives a simple geometric picture of mirror symmetry, explicitly constructing the mirror $Y$ from the quantum moduli space of objects on $X$. The duality that relates string theory on a circle $S^{1}$ of radius $R$ to a string theory on a dual circle $\hat{S}^{1}$ of radius $1 / R$ (or a product of circles) is a very basic example of a duality in string theory, called $T$-duality. Here, we see that mirror symmetry is simply $T$-duality, applied fiberwise over each point in $B$. For a review of SYZ conjecture, see [40].
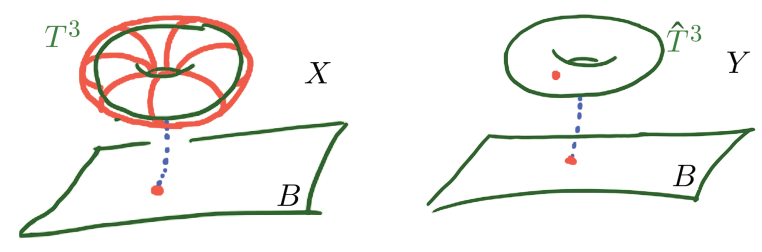

FigURE 6. SYZ mirror symmetry

One can extend the SYZ conjecture away from compact Calabi-Yau manifolds. When $X$ is a toric Calabi-Yau manifold, it is non-compact, and then the $T^{3}$ fibration is replaced by an $T^{2} \times \mathbb{R}$ fibration over $B=\mathbb{R}^{3}$. The geometry of the toric Calabi-Yau is, from this perspective, encoded in the geometry of a trivalent graph $\Gamma$ in $B$ over which the $T^{2}$ fiber degenerates to $S^{1}$. The role of $L_{p}=T^{3}$ in the compact case is replaced by $L_{p}=S^{1} \times \mathbb{R}^{2}$ : the generic $T^{2} \times \mathbb{R}$ fiber degenerates over $\Gamma$ to a union of two copies of $L_{p}$, and we take one of them. The classical moduli space of $L_{p}$ is the graph $\Gamma$; the moduli of the flat connection on $L_{p}$ is a circle fibered over this, and one still has to take into account disk instanton corrections. The quantum moduli space is a Riemann surface

$$
H_{\Gamma}(x, p)=0, \quad x, p \in \mathbb{C}^{*} .
$$

The mirror Calabi-Yau is a hypersurface

$$
Y: u v-H_{\Gamma}(x, p)=0, \quad u, v \in \mathbb{C} .
$$

The mirror to $L_{p}$ is no longer a structure sheaf on $Y$ but instead a sheaf supported on a curve, which corresponds to choosing a point on the mirror Riemannn surface 
(16) and picking either $u=0$ or $v=0$, depending on which component of the reducible $T^{2} \times \mathbb{R}$ fiber we took.

Now, let us describe what this has to do with knot theory.

4.2. Large $N$ duality, SYZ mirror symmetry, and knot theory. Large $N$ duality relates $S U(N)$ Chern-Simons theory on $S^{3}$ to Gromov-Witten theory (or an A-model topological string) on $X_{\mathbb{P}^{1}}$; this is a non-compact, toric Calabi-Yau manifold. We can obtain its mirror by application of SYZ mirror symmetry, by finding the quantum moduli space of a Lagrangian in $X_{\mathbb{P}^{1}}$ of topology of $\mathbb{R}^{2} \times S^{1}$.

In fact, we get one such Lagrangian for every knot $K$ in $S^{3}$ and with it a distinct mirror $Y_{K}$ [41]. To construct a Lagrangian $L_{K}$ in $X_{\mathbb{P}^{1}}$ of topology of $\mathbb{R}^{2} \times S^{1}$ corresponding to a knot $K$ in $S^{3}$, one starts with a Lagrangian in $X_{S^{3}}=T^{*} S^{3}$, which is a total space of the conormal bundle to the knot $K$ in the $S^{3}$ base, lifts it off the zero section (so that it does not intersect the singular locus when the $S^{3}$ shrinks), and then pushes it through the transition that relates $X_{S^{3}}$ and $X_{\mathbb{P}^{1}}$. The mirror depends only on the homotopy type of the knot:

$$
Y_{K}: u v-H_{K}(x, p)=0 .
$$

The quantum moduli space of $L_{K}$ in $X_{\mathbb{P}^{1}}$ is the Riemann surface

$$
H_{K}(x, p)=0 .
$$

The pair $x$ and $p=p_{K}(x)$ that lie on (19) are determined by summing holomorphic disks with boundaries on $L_{K}$. Large $N$ duality (14) in turn relates this to a limit of corresponding Chern-Simons amplitude:

$$
\log p_{K}(x)=x \frac{d}{d x} \lim _{\lambda \rightarrow 0} \lambda\left\langle\mathcal{O}_{K}(x)\right\rangle,
$$

where one takes $U=x$ to be a rank 1 matrix, and $Q=\exp (-t)$. For example, taking the knot $K$ to be the unknot, one gets the "conventional" mirror of $X_{\mathbb{P}^{1}}$, where

$$
H_{\bigcirc}(x, p)=1-x-p+Q x p .
$$

But, taking $K$ to be a trefoil knot instead, as an example, we get a different answer:

$$
H_{K}(x, p)=1-Q p+\left(p^{3}-p^{4}+2 p^{5}-Q p^{6}+Q^{2} p^{7}\right) x-\left(p^{9}-p^{10}\right) x^{2} .
$$

Thus, the combination of two string dualities, large $N$ duality, and mirror symmetry gives rise to a new knot invariant, the mirror Calabi-Yau manifold $Y_{K}$. Chern-Simons theory produces an infinite list of knot invariants, differing by the representations coloring the knot. To tell knots apart, it is necessary, though maybe not sufficient, to compare the entries of this list. String duality suggests that one can replace the entire list with a single invariant, the mirror Calabi-Yau manifold $Y_{K}$, plus presumably a finite set of data needed to define the quantization in this setting. Once the quantization procedure is defined, the topological B-model string is a functor that associates to $Y_{K}$ quantum invariants. Moreover, unlike knots, Calabi-Yau manifolds are easy to tell apart by simply comparing the polynomials $H_{K}(x, p)$. Thus, instead of quantum physics playing the central role in constructing good knot invariants, classical geometry of $Y_{K}$ becomes the key.

The Riemann surface $H_{K}(x, p)=0$ turns out to have an alternative mathematical formulation, as the augmentation variety of the knot [42. This is one of the knot invariants that arise from knot contact homology. Knot contact homology is the open version, developed by Lenhard $\mathrm{Ng}$ [43, of the symplectic field theory 
approach to counting holomorphic curves, pioneered by Eliashberg and Givental. This provides a relation between two distinct approaches to counting holomorphic curves, one coming from Gromov-Witten theory and the other from symplectic field theory.

\section{M-THEORY AND HOMOLOGICAL KNOT INVARIANTS}

There is a mysterious aspect of Chern-Simons knot invariants. From the definition of the Jones polynomial $J_{K}(q)$, one can see that it is always a Laurent polynomial in $q^{1 / 2}$ with integer coefficients. Coefficients of the knot polynomials are always integers, as if they are counting something. What are they counting? Since $q=e^{i \lambda}$, where $\lambda$ is either the Chern-Simons or the topological string coupling constant, the answer to this question cannot come from Chern-Simons theory or topological string theory.

Khovanov made this structure manifest in a remarkable way. He constructed a bigraded homology theory in such a way that the Jones polynomial arises as the Euler characteristic

$$
J_{K}(q)=\sum_{i, j}(-1)^{j} q^{i / 2} \operatorname{dim} \mathcal{H}_{i, j}(K),
$$

counting dimensions of knot homology groups,

$$
\mathcal{H}_{i, j}(K) \text {, }
$$

with signs. The Poincaré polynomial of knot homology

$$
P_{K}(q, t)=\sum_{i, j} t^{j} q^{i / 2} \operatorname{dim} \mathcal{H}_{i, j}(K)
$$

has strictly more information about the knot; it is a better knot invariant. One expects that this should have generalizations to all Chern-Simons (Witten-Reshetikhin-Turaev) knot and three-manifold invariants; however, knot homology theories are extremely complicated. A unified approach to categorification of quantum group invariants was very recently put forward in [45, 46]. As far as we are aware, a fully combinatorial construction of knot homologies is available only for the Jones polynomial itself.

Knot homologies have a physical interpretation within M-theory [47, 48, due to Gukov, Vafa, and Schwarz, and later Witten. Knot homologies are Hilbert spaces of states which preserve some supersymmetry in M-theory realization of ChernSimons theory. To obtain Chern-Simons theory from M-theory, one uses a similar geometry as in topological string theory. Witten was able to reduce the M-theory construction to computing cohomologies of spaces of solutions to a certain equation, the Kapustin-Witten equation [48,49] with boundary conditions depending on the knot type, but its mathematics and physics are still comparably complex. It was shown in 50] that the approach of 48 leads to the Jones polynomial once one computes the Euler characteristic. However, this has yet to lead to an explicit construction of knot homologies and $P_{K}(q, t)$, even in examples.

Physics does provide a powerful insight, if one restricts to three-manifolds and knots respecting a certain circle symmetry. In the presence of the extra symmetry, one can formulate, using M-theory, a three-dimensional topological theory, the refined Chern-Simons theory [51]. The partition function of the refined ChernSimons theory (conjecturally) computes a two-variable polynomial $I_{K}(q, t)$, a close 
cousin of Poincaré polynomial of homology theory categorifying Chern-Simons theory. For three-manifolds and knots admitting a (semifree) circle action, knot homologies corresponding to arbitrary ADE gauge groups and their representations should admit an additional grade:

$$
\mathcal{H}_{i, j}(K)=\bigoplus_{k} \mathcal{H}_{i, j, k}(K)
$$

This leads to an index, more refined than the Euler characteristic:

$$
I_{K}(q, t)=\sum_{i, j, k}(-1)^{k} q^{i / 2} t^{j+k} \operatorname{dim} \mathcal{H}_{i, j, k}(K),
$$

akin to the Hirzebruch $\chi_{y}$ genus. Setting $t=-1$, both $P_{K}(q, t)$ and $I_{K}(q, t)$ reduce to Chern-Simons invariants.

The refined Chern-Simons theory, which computes $I_{K}$, is solvable explicitly. As in Witten's solution of the "ordinary" Chern-Simons theory-by cutting the three-manifold into pieces, solving the theory on pieces, and gluing - one reduces the problem of computing the knot and three-manifold invariants to matrix multiplication. In fact, since refined Chern-Simons theory exists for a restricted set of three-manifolds and knots (those admitting a circle symmetry), a smaller set of ingredients enter; all one needs are the $S$ and the $T$ matrices providing a representation of $S L(2, Z)$ on the Hilbert space $\mathcal{H}_{T^{2}}$. The $S$ and $T$ matrices now depend on both $q$ and $t$ (they are given in Macdonald polynomials of the corresponding ADE group, evaluated at a special point, generalizing the Schur polynomials in Chern-Simons case). This is immeasurably simpler than constructions of homologies themselves. Even better, for simple representations of $S U(N)$, at large $N$ (corresponding to categorification of the HOMFLY polynomial) the index $I_{K}$ and the Poincaré polynomial $P_{K}$ of knot homology theory agree. This gives strong evidence that refined Chern-Simons theory indeed computes a new genus on knot homologies, and also evidence that M-theory is indeed behind knot homologies.

It is striking that, even though the refined Chern-Simons theory has been formulated only recently, many connections have already been made. It is known that refined Chern-Simons invariants are related to $q$-deformation of conformal blocks of $W$-algebras [52]; they have deep connections to the K-theory of the Hilbert scheme of points on $\mathbb{C}^{2}[53$ 55]. The knot invariants arising from refined Chern-Simons theory have a direct connection to representation theory of Double Affine Hecke Algebras (DAHA) [55. There is evidence that the invariants are also related to Donaldson-Thomas invariants of toric threefolds constructed recently in [56].

\section{Outlook}

Despite the successes of string theory in solving difficult problems in mathematics, this is no doubt just the tip of the iceberg. All string theories are unified in a single theory, M-theory. Genus by genus expansion, on which topological string and superstring theories are based, exists only at the corners of M-theory parameter space. Dualities fill in the rest of the diagram. M-theory has already made an appearance in knot theory context and in relating Gromov-Witten theory to Donaldson-Thomas theory.

Mathematical consequences of dualities in M-theory are largely unexplored. Two topological string theories, the A- and the B-models, with their many mathematical uses, capture supersymmetric M-theory partition functions in a very specific 


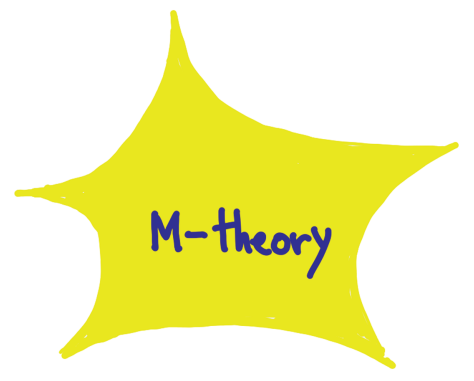

FiguRE 7. M-theory is believed to be the unique quantum theory underlying all string theories. Different descriptions of it, which emerge at the corners of the diagram, are related by dualities.

background [57,58]. The plethora of mathematical predictions extracted from topological string theory and its dualities, such as mirror symmetry and large $N$ duality, provide just a glimpse of the mathematical content of M-theory. Supersymmetric partition functions of M-theory are generalizations of topological string theory, yet only their exploration has only just begun; see [56].

To be sure, dualities do not require string theory. There are examples of dualities in quantum gauge theories which can be stated without invoking string theory. Even so, string theory often plays a crucial role in discovering the dualities, and in studying them. Symplectic duality 62, which plays an important role in knot theory and other areas of mathematics, is a duality of quantum gauge theories in three dimensions. Even though today one can phrase it purely in gauge theory language, the duality was discovered using string theory, in 59, 60, and string theory helps one understand it better [61. Seiberg-Witten $(S W)$ theory, the celebrated 4d QFT with an important role for four-manifold invariants [63, 64, turns out to have many dual descriptions [65]. In fact most of the theories in this class turn out not have a conventional description but need M-theory for their definition. To define them, one considers the six-dimensional "theory $X$ " that arises as a part of M-theory, compactified on a Riemann surface $C$. Only in certain corners of the moduli of $C$ does the usual gauge theory description emerge. This observation leads to a precise mathematical prediction: the partition functions of this class of Seiberg-Witten theories are the conformal blocks on $C$ of a class of two-dimensional conformal field theories with $W$-algebra symmetry [66. This unifies problems in QFT, geometry, and representation theory. Some aspects of this correspondence were recently proven in [67. The $S$-duality of four-dimensional $\mathcal{N}=4$ Yang-Mills theory, related to electric-magnetic duality, is believed to be the duality underlying the geometric Langlands program 68 71. The Langlands program has, for the last 50 years, been one of the key unifying themes in mathematics [72]. Once again, even though one can phrase $S$ duality in terms of gauge theory alone, much of our understanding of it comes from string theory: four-dimensional $\mathcal{N}=4$ Yang-Mills theory arises by compactifying the six-dimensional theory $X$ on a torus, and $S$-duality simply comes from $S L(2, Z)$ symmetry of the torus!

The interacting between the two fields has only really begun in earnest. It is fairly certain that dualities in string theory and quantum field theory hold potential for many new breakthroughs in mathematics by extracting their mathematical 
predictions and proving them. It should also lead to a deeper and sharper understanding of quantum physics. There is a good chance that eventually our view of mathematics and quantum physics will have changed profoundly.

\section{ACKNOWLEDGMENTS}

I am grateful to Robbert Dijkgraaf, Tobias Ekholm, Nathan Haouzi, Albrecht Klemm, Markos Marino, Lenhard Ng, Shamil Shakirov, Andrei Okounkov, and Cumrun Vafa for years of collaborations that taught me the ideas presented, and to the numerous mathematicians and physicists who have worked together to unearth the beautiful structures in our subject.

\section{About THE AUthor}

Mina Aganagic is professor of mathematics and physics at UC Berkeley.

\section{REFERENCES}

[1] J. Polchinski, String duality: A colloquium, Rev. Mod. Phys. 68 (1996), 1245 [hepth/9607050].

[2] C. Vafa, Geometric physics, hep-th/9810149.

[3] J. Polchinski, Dualities of Fields and Strings, arXiv:1412.5704 [hep-th].

[4] G. Moore, Physical Mathematics and the Future, http://www.physics.rutgers.edu/gmoore/ PhysicalMathematicsAndFuture.pdf

[5] V. F. R. Jones, A polynomial invariant for knots via von Neumann algebras, Bull. Amer. Math. Soc. (N.S.) 12 (1985), no. 1, 103-111, DOI 10.1090/S0273-0979-1985-153042. MR766964 (86e:57006)

[6] V. F. R. Jones, On knot invariants related to some statistical mechanical models, Pacific J. Math. 137 (1989), no. 2, 311-334. MR990215 (89m:57005)

[7] Edward Witten, Quantum field theory and the Jones polynomial, Comm. Math. Phys. 121 (1989), no. 3, 351-399. MR990772 (90h:57009)

[8] N. Reshetikhin and V. G. Turaev, Invariants of 3-manifolds via link polynomials and quantum groups, Invent. Math. 103 (1991), no. 3, 547-597, DOI 10.1007/BF01239527. MR1091619 (92b:57024)

[9] Kentaro Hori, Sheldon Katz, Albrecht Klemm, Rahul Pandharipande, Richard Thomas, Cumrun Vafa, Ravi Vakil, and Eric Zaslow, Mirror symmetry, Clay Mathematics Monographs, vol. 1, American Mathematical Society, Providence, RI; Clay Mathematics Institute, Cambridge, MA, 2003. With a preface by Vafa. MR2003030 (2004g:14042)

[10] Andrei Okounkov, Random surfaces enumerating algebraic curves, European Congress of Mathematics, Eur. Math. Soc., Zürich, 2005, pp. 751-768. MR2185779 (2007b:14122)

[11] R. Thomas, "The geometry of mirror symmetry", Encylopedia of mathematical physics. Francoise, Naber, and Tsou (editors), Elsevier, Oxford.

[12] Edward Witten, Topological sigma models, Comm. Math. Phys. 118 (1988), no. 3, 411-449. MR.958805 (90b:81080)

[13] Edward Witten, On the structure of the topological phase of two-dimensional gravity, Nuclear Phys. B 340 (1990), no. 2-3, 281-332, DOI 10.1016/0550-3213(90)90449-N. MR.1068086 (91m:32020)

[14] Edward Witten, Mirror manifolds and topological field theory, Essays on mirror manifolds, Int. Press, Hong Kong, 1992, pp. 120-158. MR1191422 (94c:81194)

[15] M. Bershadsky, S. Cecotti, H. Ooguri, and C. Vafa, Kodaira-Spencer theory of gravity and exact results for quantum string amplitudes, Comm. Math. Phys. 165 (1994), no. 2, 311-427. MR.1301851 (95f:32029)

[16] Wolfgang Lerche, Cumrun Vafa, and Nicholas P. Warner, Chiral rings in $N=2$ superconformal theories, Nuclear Phys. B 324 (1989), no. 2, 427-474, DOI 10.1016/0550-3213(89)904744. MR1025424(91d:81132) 
[17] Philip Candelas, Xenia C. de la Ossa, Paul S. Green, and Linda Parkes, A pair of Calabi-Yau manifolds as an exactly soluble superconformal theory, Nuclear Phys. B 359 (1991), no. 1, 21-74, DOI 10.1016/0550-3213(91)90292-6. MR1115626 (93b:32029)

[18] K. J. Costello and S. Li, "Quantum BCOV theory on Calabi-Yau manifolds and the higher genus B-model," arXiv:1201.4501 [math.QA].

[19] E. Witten, Chern-Simons gauge theory as a string theory, The Floer memorial volume, Progr. Math., vol. 133, Birkhäuser, Basel, 1995, pp. 637-678. MR1362846 (97j:57052)

[20] Rajesh Gopakumar and Cumrun Vafa, On the gauge theory/geometry correspondence, Adv. Theor. Math. Phys. 3 (1999), no. 5, 1415-1443. MR.1796682(2001k:81272)

[21] C. Faber and R. Pandharipande, Hodge integrals and Gromov-Witten theory, Invent. Math. 139 (2000), no. 1, 173-199, DOI 10.1007/s002229900028. MR1728879 (2000m:14057)

[22] Hirosi Ooguri and Cumrun Vafa, Knot invariants and topological strings, Nuclear Phys. B 577 (2000), no. 3, 419-438, DOI 10.1016/S0550-3213(00)00118-8. MR 1765411(2001i:81254)

[23] Clifford Henry Taubes, Lagrangians for the Gopakumar-Vafa conjecture, Adv. Theor. Math. Phys. 5 (2001), no. 1, 139-163. MR1894340 (2003f:53163)

[24] Dror Bar-Natan, On the Vassiliev knot invariants, Topology 34 (1995), no. 2, 423-472, DOI 10.1016/0040-9383(95)93237-2. MR1318886 (97d:57004)

[25] Marcos Mariño, Chern-Simons theory, matrix integrals, and perturbative three-manifold invariants, Comm. Math. Phys. 253 (2005), no. 1, 25-49, DOI 10.1007/s00220-004-1194-4. MR2105636(2005k:81290)

[26] K. Fukaya, Talk at String-Math 2013, http : //media.scgp.stonybrook.edu/presentations/ 201306182 fukaya.pdf

[27] Sheldon Katz and Chiu-Chu Melissa Liu, Enumerative geometry of stable maps with Lagrangian boundary conditions and multiple covers of the disc, Adv. Theor. Math. Phys. 5 (2001), no. 1, 1-49. MR.1894336 (2003e:14047)

[28] D. Maulik, A. Oblomkov, A. Okounkov, and R. Pandharipande, Gromov-Witten/DonaldsonThomas correspondence for toric 3-folds, Invent. Math. 186 (2011), no. 2, 435-479, DOI 10.1007/s00222-011-0322-y. MR2845622(2012h:14140)

[29] G. 't Hooft, "A planar diagram theory for strong interactions," Nucl. Phys. B 72, 461 (1974).

[30] Mina Aganagic, Albrecht Klemm, Marcos Mariño, and Cumrun Vafa, The topological vertex, Comm. Math. Phys. 254 (2005), no. 2, 425-478, DOI 10.1007/s00220-004-1162-z. MR2117633 (2006e:81263)

[31] S. K. Donaldson and R. P. Thomas, Gauge theory in higher dimensions, The geometric universe (Oxford, 1996), Oxford Univ. Press, Oxford, 1998, pp. 31-47. MR.1634503(2000a:57085)

[32] D. Maulik, N. Nekrasov, A. Okounkov, and R. Pandharipande, Gromov-Witten theory and Donaldson-Thomas theory. I, Compos. Math. 142 (2006), no. 5, 1263-1285, DOI 10.1112/S0010437X06002302. MR2264664 (2007i:14061)

[33] D. Maulik, N. Nekrasov, A. Okounkov, and R. Pandharipande, Gromov-Witten theory and Donaldson-Thomas theory. II, Compos. Math. 142 (2006), no. 5, 1286-1304, DOI 10.1112/S0010437X06002314. MR2264665 (2007i:14062)

[34] Andrei Okounkov, Nikolai Reshetikhin, and Cumrun Vafa, Quantum Calabi-Yau and classical crystals, The unity of mathematics, Progr. Math., vol. 244, Birkhäuser Boston, Boston, MA, 2006, pp. 597-618, DOI 10.1007/0-8176-4467-9_16. MR2181817 (2006k:81342)

[35] Amer Iqbal, Cumrun Vafa, Nikita Nekrasov, and Andrei Okounkov, Quantum foam and topological strings, J. High Energy Phys. 4 (2008), 011, 47, DOI 10.1088/1126-6708/2008/04/011. MR 2425292(2009k:81210)

[36] R. Dijkgraaf, C. Vafa and E. Verlinde, "M-theory and a topological string duality," hepth/0602087.

[37] Maxim Kontsevich, Homological algebra of mirror symmetry, Proceedings of the International Congress of Mathematicians, Vol. 1, 2 (Zürich, 1994), Birkhäuser, Basel, 1995, pp. 120-139. MR:1403918 (97f:32040)

[38] Yuichi Nohara and Kazushi Ueda, Homological mirror symmetry for the quintic 3-fold, Geom. Topol. 16 (2012), no. 4, 1967-2001, DOI 10.2140/gt.2012.16.1967. MR.2975297

[39] Andrew Strominger, Shing-Tung Yau, and Eric Zaslow, Mirror symmetry is T-duality, Nuclear Phys. B 479 (1996), no. 1-2, 243-259, DOI 10.1016/0550-3213(96)00434-8. MR.1429831 (97j:32022)

[40] Mark Gross, Mirror symmetry and the Strominger-Yau-Zaslow conjecture, Current developments in mathematics 2012, Int. Press, Somerville, MA, 2013, pp. 133-191. MR 3204345 
[41] M. Aganagic and C. Vafa, "Large N Duality, Mirror Symmetry, and a Q-deformed Apolynomial for Knots," arXiv:1204.4709;

[42] Mina Aganagic, Tobias Ekholm, Lenhard Ng, and Cumrun Vafa, Topological strings, D-model, and knot contact homology, Adv. Theor. Math. Phys. 18 (2014), no. 4, 827-956. MR3277674

[43] Lenhard Ng, Combinatorial knot contact homology and transverse knots, Adv. Math. 227 (2011), no. 6, 2189-2219, DOI 10.1016/j.aim.2011.04.014. MR2807087(2012j:53116)

[44] Mikhail Khovanov, A categorification of the Jones polynomial, Duke Math. J. 101 (2000), no. 3, 359-426, DOI 10.1215/S0012-7094-00-10131-7. MR.1740682 (2002j:57025)

[45] Webster, B., "Knot invariants and higher representation theory" arXiv:1309.3796

[46] Webster, B., "Tensor product algebras, Grassmannians and Khovanov homology" arXiv:1312.7357

[47] Sergei Gukov, Albert Schwarz, and Cumrun Vafa, Khovanov-Rozansky homology and topological strings, Lett. Math. Phys. 74 (2005), no. 1, 53-74, DOI 10.1007/s11005-005-0008-8. MR2193547(2007a:57014)

[48] Edward Witten, Fivebranes and knots, Quantum Topol. 3 (2012), no. 1, 1-137, DOI 10.4171/QT/26. MR 2852941

[49] Edward Witten, Khovanov homology and gauge theory, Proceedings of the Freedman Fest, Geom. Topol. Monogr., vol. 18, Geom. Topol. Publ., Coventry, 2012, pp. 291-308, DOI 10.2140/gtm.2012.18.291. MR3084242

[50] Davide Gaiotto and Edward Witten, Knot invariants from four-dimensional gauge theory, Adv. Theor. Math. Phys. 16 (2012), no. 3, 935-1086. MR.3024278

[51] M. Aganagic and S. Shakirov, "Knot Homology from Refined Chern-Simons Theory," arXiv:1105.5117 [hep-th].

[52] M. Aganagic, N. Haouzi and S. Shakirov, " $A_{n}$-Triality," arXiv:1403.3657 [hep-th].

[53] Oblomkov, A., Rasmussen, J., \& Shende, V., "The Hilbert scheme of a plane curve singularity and the HOMFLY homology of its link", arXiv:1201.2115

[54] Hiraku Nakajima, Refined Chern-Simons theory and Hilbert schemes of points on the plane, Perspectives in representation theory, Contemp. Math., vol. 610, Amer. Math. Soc., Providence, RI, 2014, pp. 305-331, DOI 10.1090/conm/610/12157. MR3220632

[55] Gorsky, E., \& Negut, A., "Refined Knot Invariants and Hilbert Schemes", arXiv:1304.3328

[56] N. Nekrasov and A. Okounkov, "Membranes and Sheaves," arXiv:1404.2323 [math.AG].

[57] R. Gopakumar and C. Vafa, "M theory and topological strings. 1.," hep-th/9809187.

[58] R. Gopakumar and C. Vafa, "M theory and topological strings. 2.," hep-th/9812127.

[59] Amihay Hanany and Edward Witten, Type IIB superstrings, BPS monopoles, and threedimensional gauge dynamics, Nuclear Phys. B 492 (1997), no. 1-2, 152-190, DOI 10.1016/S0550-3213(97)00157-0. MR 1451054(98h:81096)

[60] K. Intriligator and N. Seiberg, Mirror symmetry in three-dimensional gauge theories, Phys. Lett. B 387 (1996), no. 3, 513-519, DOI 10.1016/0370-2693(96)01088-X. MR1413696 $(97 \mathrm{j}: 81261)$

[61] Davide Gaiotto and Edward Witten, Supersymmetric boundary conditions in $\mathcal{N}=4$ super Yang-Mills theory, J. Stat. Phys. 135 (2009), no. 5-6, 789-855, DOI 10.1007/s10955-0099687-3. MR 2548595 (2011a:81238)

[62] Tom Braden, Nicholas Proudfoot, Ben Webster: "Quantizations of conical symplectic resolutions I: local and global structure", arXiv:1208.3863; "Quantizations of conical symplectic resolutions II: category $\mathcal{O}$ and symplectic duality", arXiv: 1407.0964

[63] N. Seiberg and E. Witten, Erratum: "Electric-magnetic duality, monopole condensation, and confinement in $N=2$ supersymmetric Yang-Mills theory", Nuclear Phys. B 430 (1994), no. 2, 485-486, DOI 10.1016/0550-3213(94)00449-8. MR1303306 (95m:81202b)

[64] Edward Witten, Monopoles and four-manifolds, Math. Res. Lett. 1 (1994), no. 6, 769-796, DOI 10.4310/MRL.1994.v1.n6.a13. MR1306021 (96d:57035)

[65] Davide Gaiotto, $N=2$ dualities, J. High Energy Phys. 8 (2012), 034, front matter +57. MR.3006961

[66] Luis F. Alday, Davide Gaiotto, and Yuji Tachikawa, Liouville correlation functions from four-dimensional gauge theories, Lett. Math. Phys. 91 (2010), no. 2, 167-197, DOI 10.1007/s11005-010-0369-5. MR2586871 (2010k:81243)

[67] A. Braverman, M. Finkelberg, H. Nakajima, "Instanton moduli spaces and W-algebras", arxiv:1406.2381[math:QA] 
[68] C. Montonen and D. Olive, Phys. Lett. B72 (1977) 117; P. Goddard, J. Nyuts and D. Olive, Nucl. Phys. B125 (1977) 1.

[69] Cumrun Vafa and Edward Witten, A strong coupling test of S-duality, Nuclear Phys. B 431 (1994), no. 1-2, 3-77, DOI 10.1016/0550-3213(94)90097-3. MR1305096 (95k:81138)

[70] Anton Kapustin and Edward Witten, Electric-magnetic duality and the geometric Langlands program, Commun. Number Theory Phys. 1 (2007), no. 1, 1-236, DOI 10.4310/CNTP.2007.v1.n1.a1. MR2306566 (2008g:14018)

[71] R. Donagi and T. Pantev, Langlands duality for Hitchin systems, Invent. Math. 189 (2012), no. 3, 653-735, DOI 10.1007/s00222-012-0373-8. MR2957305

[72] E. Frenkel, "Lectures on the Langlands program and conformal field theory," hep-th/0512172.

Departments of Mathematics and Physics, University of California, Berkeley, CalIFORNIA

E-mail address: aganacic@berkeley.edu 